\title{
PET/CT-Guided Percutaneous Biopsy of Isolated Intramuscular Metastases from Postcricoid Cancer
}

\author{
Govindarajan J.I. Mallarajapatna, Kumar G. Kallur, Nagaraj K. Ramanna, Sridhar P. Susheela, and \\ Prashanth G. Ramachandra
}

Department of Radiology, Bangalore Institute of Oncology and Health Care Global, Bangalore, India

\begin{abstract}
${ }^{18}$ F-FDG PET/CT has made it possible to identify many unsuspected lesions such as isolated intramuscular metastases because of its ability to detect abnormal metabolic activity in an early stage even without clinical symptoms or morphologic changes. We need to modify our routine techniques for performing biopsies of such lesions because they may not be visualized on morphologic imaging. We describe a technique in which fused $\mathrm{PET} / \mathrm{CT}$ is used to guide the percutaneous biopsy needle for sampling isolated intramuscular metastatic lesions in a patient whose intramuscular metastases were identified only on PET/ CT, not on CT or ultrasound, and were clinically occult.
\end{abstract}

Key Words: molecular imaging; PET/CT; biopsy; metastases

J Nucl Med Technol 2009; 37:220-222

DOI: 10.2967/jnmt.109.064709

W e report a patient in whom intramuscular metastases were identified on PET/CT but not seen on CT or ultrasound and were clinically occult and, hence, in whom we used PET/CT to guide the percutaneous biopsy needle for sampling the lesion.

\section{CASE REPORT}

A 35-y-old woman presented with difficulty in swallowing. Esophagoscopy revealed a large mass with significant narrowing posterior to the cricoid region. The scope was not negotiable beyond the stricture. Biopsy samples from the mass had revealed squamous cell carcinoma.

The patient was referred to our department for a staging PET/CT scan, which was performed with a 16-slice PET/ CT scanner (GE Healthcare) after intravenous injection of $370 \mathrm{MBq}$ of ${ }^{18} \mathrm{~F}-\mathrm{FDG}$. A diagnostic CT scan of the neck, chest, and abdomen with intravenous iodine contrast administration was also performed.

The mass at the postcricoid region was well delineated and appeared to extend into the thoracic esophagus and left tracheoesophageal groove, causing left vocal cord palsy.

Received Mar. 30, 2009; revision accepted Aug. 3, 2009.

For correspondence or reprints contact: M.J. Govindarajan, No. 6, 3rd cross, LIC colony, Basaveshwar Nagar, Bangalore, 560079 India.

E-mail: revathigovind@gmail.com

COPYRIGHT @ 2009 by the Society of Nuclear Medicine, Inc.
There were foci of increased ${ }^{18} \mathrm{~F}-\mathrm{FDG}$ accumulation within the skeletal muscles of the right lateral chest wall and axilla and the erector spinae muscles (Fig. 1). These were not apparent on contrast-enhanced CT images. A few lower neck and mediastinal metastatic nodes were present. No other metastases were identified. MRI was not performed.

A probable diagnosis of skeletal muscle metastases from postcricoid esophageal cancer was made. An image-guided biopsy of the intramuscular lesions was planned.

The lesions were not well appreciated on high-frequency ultrasound. Because the lesions were also not visible on contrast-enhanced CT scans, ${ }^{18} \mathrm{~F}$-FDG PET/CT was again used for localization of the lesions. Fused images of CT and PET were obtained, and a biopsy sample was obtained of one of the lesions in the left erector spinae muscle (Fig. 2). CT images with the needle in place were fused with PET images to accurately position the needle into the metabolically active lesion. The diagnosis of metastasis was established.

\section{DISCUSSION}

The use of ${ }^{18} \mathrm{~F}-\mathrm{FDG}$ PET/CT for biopsy guidance is a new technique, emerging after PET/CT became established in clinical practice.

There are reports of PET/CT being used for guiding percutaneous biopsy procedures because of the dual information it provides about morphology and physiology. It has been used for percutaneous puncture of an infected cyst in a patient with autosomal-dominant polycystic kidney disease. The exact site of the infected cyst could be demonstrated by the PET study. PET/CT has been used to localize the electrode tip during radiofrequency ablation of hepatic metastases. In previously treated lesions, PET/CT can differentiate between viable and necrotic areas and guide biopsy and other interventions (1-3). PET/CT has been reported to be of use in fine-needle aspiration cytology of lung masses (4). Logically, with the incorporation of morphologic details from CT and MRI and functional details from PET, the ability to guide intervention should improve considerably $(5,6)$.

PET imaging with the needle in place during a biopsy is impractical because of the long time needed to acquire the images at the current technical standard and because the 
A

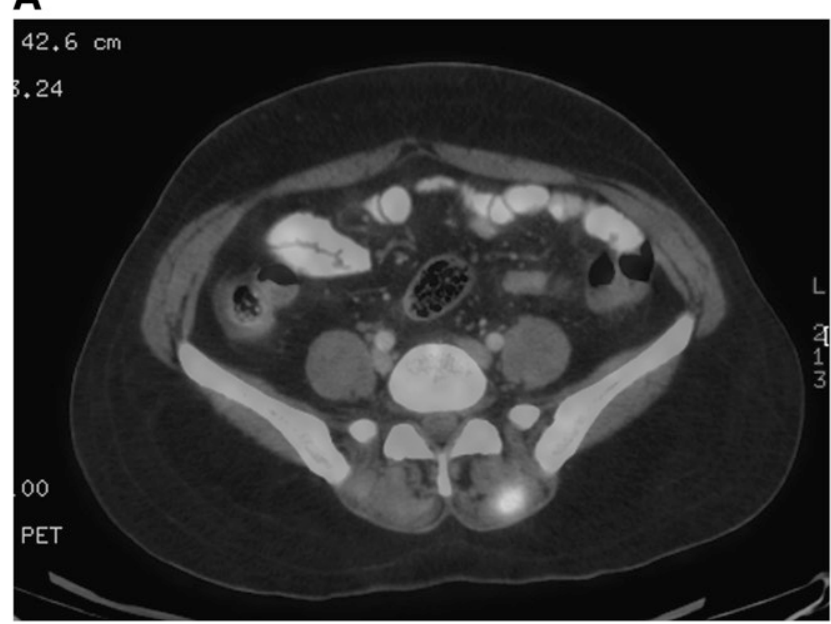

B

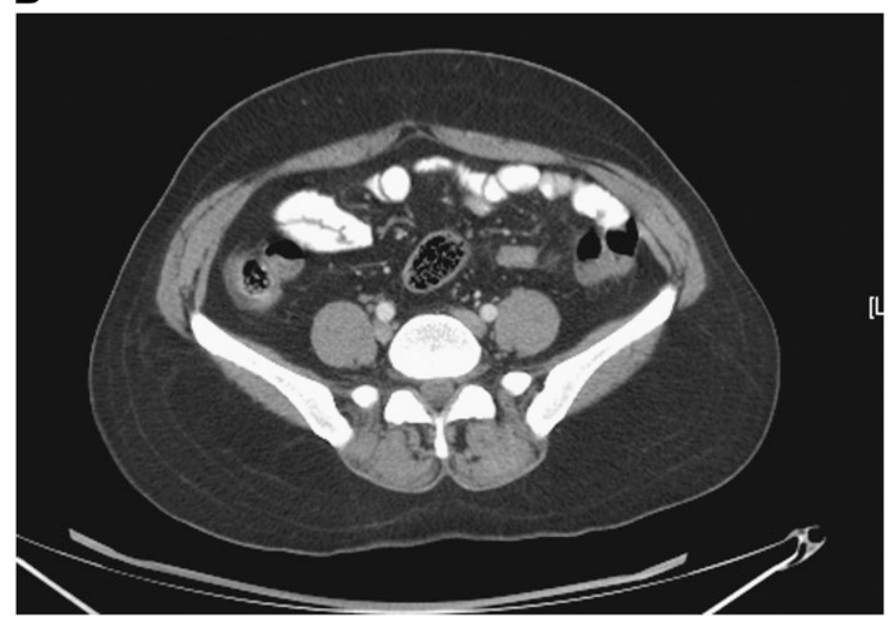

FIGURE 1. (A) Fused PET/CT image demonstrating erector spinae muscle deposits, more intense on left side. (B) Contrastenhanced CT scan at same level as in A, not showing any definite pathology in erector spinae muscles.

needle will never be easily identifiable on source PET images. The PET/CT scan may therefore be used for percutaneous biopsy in only 2 ways: a preprocedural $\mathrm{PET} / \mathrm{CT}$ scan in which biopsy is performed only under CT guidance after the lesion has been identified on the fused PET/CT image, and fusion of a CT image acquired with the needle in place and a PET image acquired before needle insertion. The second method is more accurate because the metabolically active focus can be determined with respect to the needle tip. We followed the second method in our patient.

There are a few theoretic drawbacks to this technique. First, each time the needle is advanced and CT is performed, an additional interval is required to fuse the previously acquired PET image with the CT image. Second, the lead shield used to reduce radiation to the operator limits the operator's movements. Finally, the operator and the supporting staff do receive an additional radiation dose, however small it may be. Yet, with experience, we have acquired the skill of performing this type of biopsy without spending much time with the patient. Also, our experience is that the lead shield has not significantly limited our movements during the procedure.

We have tried to calculate the extra radiation dose to the operator by using a pocket dosimeter while performing the procedure. We usually call the patient to return for a delayed scan after we have reviewed the results of the diagnostic PET/CT scan. Therefore, the delayed scan will generally take place about 5-6 h after injection of the radiopharmaceutical for the diagnostic scan. Because of decay, radioactivity will be significantly reduced, but some activity will persist at lesion sites. On average, we have recorded a surface dose to the operator of less than $1 \mu \mathrm{Sv}$ for each procedure. Assuming an average of 1 procedure per day, and about 300 working days annually, the annual dose to the operator is only an additional $300 \mu \mathrm{Sv}$, which is
A

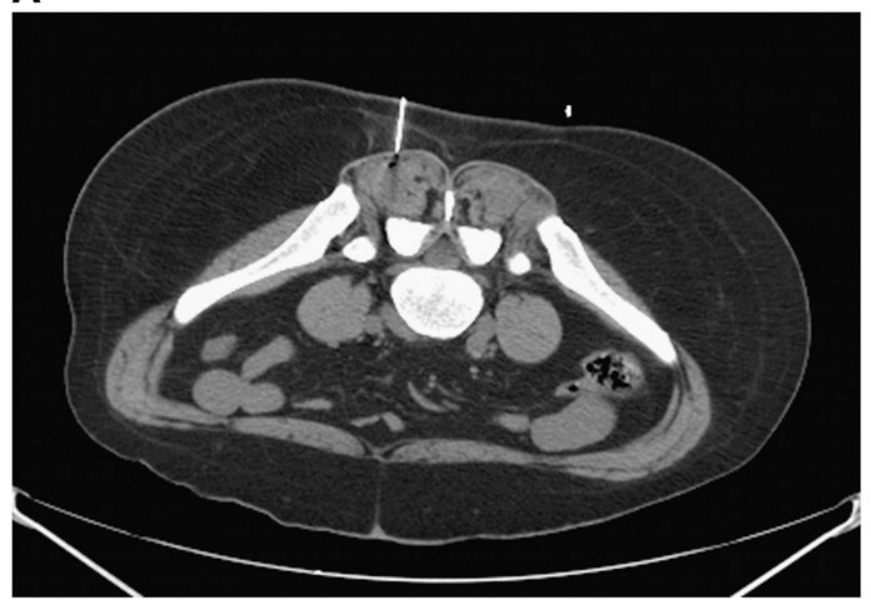

B

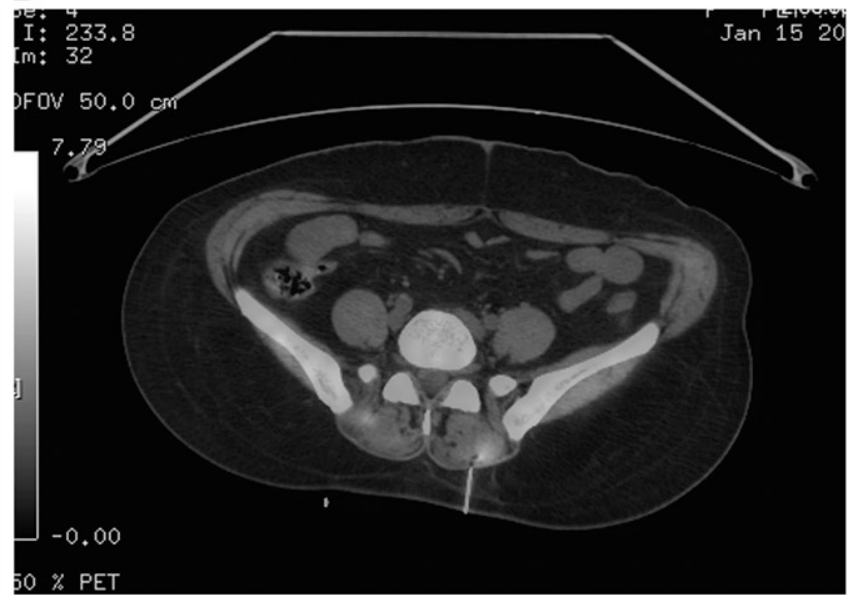

FIGURE 2. (A) CT scan showing needle in left erector spinae muscle. (B) Fusion of A with PET image to document needle in relation to lesion. 
significantly less than the recommended $0.05-\mathrm{Sv}$ annual limit. Our overall radiation exposure per day at the workplace has never exceeded $2 \mu \mathrm{Sv}$. We can conclude that the radiation dose to the operator and the supporting staff during the biopsy is insignificant, especially given the fact that we use a lead shield with a window by the side of scanner gantry while performing the procedure, further reducing the radiation dose to the operator.

However, PET/CT may have false-positive results, particularly when intramuscular lesions are found, as observed in 2 lung cancer patients with gluteal injection sites demonstrating high ${ }^{18} \mathrm{~F}$-FDG accumulation in a report by Prosch et al. (7). This false positivity is due to the high sensitivity of this modality, which also demonstrates high ${ }^{18} \mathrm{~F}$-FDG accumulation in noncancerous lesions such as some benign infectious or inflammatory pathologies. Hence, a cytologic confirmation is mandatory in suspected unusual metastases. This is particularly so in India, where tuberculosis is endemic.

In our patient, it was only because of the PET/CT scan that the intramuscular lesions were identified and then could undergo biopsy, as these were clinically occult and not apparent on ultrasound or contrast-enhanced CT. To our knowledge, this is the first report of PET/CT-guided biopsy of isolated intramuscular metastases from any cancer that was clinically occult and not apparent on other morphologic imaging modalities such as CT or ultrasound.

\section{CONCLUSION}

It is logical to use PET/CT for localizing and guiding percutaneous procedures when the lesions are clinically occult and not apparent on morphologic imaging studies. Also, PET/CT is much more helpful than morphologic imaging alone in staging cancer patients. A combination of both morphologic and functional imaging is optimal both for identifying and for performing a biopsy on lesions.

\section{REFERENCES}

1. Kaim AH, Burger C, Ganter CC, et al. PET-CT-guided percutaneous puncture of an infected cyst in autosomal dominant polycystic kidney disease: case report. Radiology. 2001;221:818-821.

2. Prior JO, Kosinski M, Delaloye AB, Denys A. Initial report of PET/CT-guided radiofrequency ablation of liver metastases. J Vasc Interv Radiol. 2007;18:801803.

3. Herzog P, Scher B, Helmberger T, Hahn K, Reiser MF, Becker CR. PET-CT interventional tumour therapy. Radiologe. 2004;44:1088-1095.

4. Govindarajan MJ, Kalyanpur A, Nagaraj KR, Ravikumar H, Kallur KG, Sridhar PS. Technical note: preprocedural PET/CT guidance for fine needle aspiration cytology of a lung mass. Indian J Radiol Imaging. 2008;18:90-91.

5. Yap JT, Camey JP, Hall NC, Townsend DW. Image-guided cancer therapy using PET/CT. Cancer J. 2004;10:221-233.

6. Solomon SB, Incorporating CT, MR imaging and positron emission tomography into minimally invasive therapies. J Vasc Interv Radiol. 2005;16:445-447.

7. Prosch H, Mirzaei S, Oschatz E, Strasser G, Huber M, Mostbeck G, Gluteal injection site granulomas: false positive finding on FDG-PET in patients with nonsmall cell lung cancer. Br J Radiol. 2005;78:758-761. 